\title{
Heat-driven spin currents on large scales
}

\section{Sylvain D. Brechet ${ }^{\star}$ and Jean-Philippe Ansermet}

Institute of Condensed Matter Physics, Station 3, Ecole Polytechnique Fédérale de Lausanne - EPFL, 1015 Lausanne, Switzerland

Received 7 April 2011, revised 8 June 2011, accepted 10 June 2011

Published online 17 June 2011

Keywords spintronics, spin currents, spin Seebeck effect

${ }^{*}$ Corresponding author: e-mail sylvain.brechet@epfl.ch

Using a three-current model (i.e. heat, spin-up and spin-down electrons) the thermodynamics of irreversible processes predicts that a temperature gradient gives rise to a spin current on large scales, in particular under the conditions used to measure the spin Seebeck effect. Diffusive currents on large scales are common in thermochemistry. The proportionality between the diffusion current and the gradient of the chemical potential is known as the Soret effect or thermophoresis.
1 Introduction Spintronics is concerned with using the spin of the electron in novel electronic devices. The spin property adds a degree of freedom to the electron transport. Recently, it has become evident that a number of studies of spin-dependent transport included also heat transport and the term "spin caloritronics" was coined [1]. While the charge, spin and heat currents were considered by spintronics founders $[2,3]$, much attraction to the inclusion of heat in spin-dependent transport stems from the discovery of the so-called spin Seebeck effect [4, 5].

The existence of a bulk spin current on large scales at distances orders of magnitude larger than the spin diffusion length may appear surprising [6], since the more familiar GMR vanishes on such scales.

From simple thermodynamical considerations, the currents are functions of the gradients of the intensive thermodynamical variables, i.e. the temperature gradient $\nabla T$, the chemical potential gradient $\nabla \mu$ and the electric potential gradient $\nabla V$, which vanish at equilibrium. In order to observe a spin polarisation current, the system has to interact with the exterior through a heat current, an electric current or both. In this Letter, we consider the first case, where a spin polarisation current is driven by a heat current in a stationary state.

We use the three-current model (spin-up, spin-down and entropy currents) to show that under the boundary conditions used in the spin Seebeck experiments, one can expect a non-vanishing spin current. The result is known in chemistry as a Soret effect, as discussed in the concluding section, below.

2 Three-current model We assume that the electric charge carriers are of two types, up spins and down spins, labeled respectively with a subscript $(+)$ and $(-)$, and considered as two different "chemical substances". Then, we use a three-current model involving a heat current density and the two charge current densities. We adopt the notation of a previous work [7]. A similar three-current model was recently used by [8]. The three diffusive currents are the entropy current density $\boldsymbol{j}_{s}$ and the electric current densities $\boldsymbol{j}_{+}$and $\boldsymbol{j}_{-}$of the spin-up and spin-down carriers. Thermodynamics of irreversible processes implies that there are linear relations between these current densities and their respective generalized forces. These relations relate the entropy current $\boldsymbol{j}_{s}$ and the charge current densities $\boldsymbol{j}_{+}$and $\boldsymbol{j}_{-}$ respectively to the gradient of temperature, $\nabla T$, and the gradients of the respective electrochemical potential of the charge carriers, $\nabla \mu_{+}$and $\nabla \mu_{-}$, according to

$$
\left(\begin{array}{c}
\boldsymbol{j}_{s} \\
\boldsymbol{j}_{+} \\
\boldsymbol{j}_{-}
\end{array}\right)=-\left(\begin{array}{lll}
\mathcal{L}_{s s} & \mathcal{L}_{s+} & \mathcal{L}_{s-} \\
\mathcal{L}_{+s} & \mathcal{L}_{++} & \mathcal{L}_{+-} \\
\mathcal{L}_{-s} & \mathcal{L}_{-+} & \mathcal{L}_{--}
\end{array}\right)\left(\begin{array}{l}
\nabla T \\
\nabla \mu_{+} \\
\nabla \mu_{-}
\end{array}\right) .
$$

For convenience, we adopt the notation of Valet and Fert [9] and express the electrochemical potentials $\mu_{ \pm}$as

$$
\mu_{ \pm}=\mu_{0} \pm \Delta \mu+q V \text {, }
$$


where $q$ is the charge per carrier, $V$ the applied electrostatic potential and $\mu_{0}$ the mean chemical potential. In our discussion, we do not consider purely chemical effects, so $\nabla \mu_{0}=0$. Moreover, the Onsager coefficients $\mathcal{L}_{+-}$and $\mathcal{L}_{-+}$ describe spin mixing [10]. These coefficients are only important when describing the magnetic field dependence of the thermoelectric power [11], and can thus be neglected in our approach

As shown by Gravier et al. [7], it is straightforward to recast the linear relations (1) as

$$
\left(\begin{array}{l}
\boldsymbol{j}_{s} \\
\boldsymbol{j}_{+} \\
\boldsymbol{j}_{-}
\end{array}\right)=-\left(\begin{array}{ccc}
\kappa & \sigma_{-} \varepsilon_{-} & \sigma_{+} \varepsilon_{+} \\
\sigma_{+} \varepsilon_{+} & \frac{\sigma_{+}}{q} & 0 \\
& 0 & \frac{\sigma_{-}}{q}
\end{array}\right)\left(\begin{array}{l}
\nabla T \\
\nabla \mu_{+} \\
\nabla \mu_{-}
\end{array}\right),
$$

where $\sigma_{ \pm}$are the spin-dependent electric conductivities of the spin currents, $\kappa$ is the thermal conductivity, and $\varepsilon_{+}$are the spin-dependent Seebeck coefficients of the spin currents, defined as

$$
\varepsilon_{ \pm}=-\frac{1}{q} \frac{\nabla \mu_{ \pm}}{\nabla T}
$$

At this point, it is physically useful to define the electric current density $\boldsymbol{j}$ and the spin polarisation current density $\boldsymbol{j}_{p}$ as the sum and the difference of the spin-dependent current densities $\boldsymbol{j}_{+}$and $\boldsymbol{j}_{-}$respectively, i.e.

$$
\boldsymbol{j}=\boldsymbol{j}_{+}+\boldsymbol{j}_{-} \quad \text { and } \quad \boldsymbol{j}_{p}=\boldsymbol{j}_{+}-\boldsymbol{j}_{-} .
$$

Similarly, we define the effective electric conductivity $\sigma$ and the spin-dependent polarisation conductivity $\sigma_{p}$

$$
\sigma=\sigma_{+}+\sigma_{-} \quad \text { and } \quad \sigma_{p}=\sigma_{+}-\sigma_{-} .
$$

In the bulk limit, the spin channels reach equilibrium, which implies that $\Delta \mu=0$ and from (2), it follows that $\nabla \mu \equiv \nabla \mu_{+}=\nabla \mu_{-}$. In this limit, we define the effective spin Seebeck coefficient $\varepsilon$ and the polarisation spin Seebeck coefficient $\varepsilon_{p}$ as

$$
\varepsilon=-\left.\frac{1}{q} \frac{\nabla \mu}{\nabla T}\right|_{j=0} \quad \text { and } \quad \varepsilon_{p}=-\left.\frac{1}{q} \frac{\nabla \mu}{\nabla T}\right|_{j_{p}=0} .
$$

The linear relations (3) imply when $\boldsymbol{j}=0$ and $\boldsymbol{j}_{p}=0$ respectively that

$$
\begin{aligned}
& \left(\sigma_{+} \varepsilon_{+}+\sigma_{-} \varepsilon_{-}\right) \nabla T+\frac{\sigma_{+}+\sigma_{-}}{q} \nabla \mu=0, \\
& \left(\sigma_{+} \varepsilon_{+}-\sigma_{-} \varepsilon_{-}\right) \nabla T+\frac{\sigma_{+}-\sigma_{-}}{q} \nabla \mu=0 .
\end{aligned}
$$

By comparing the relations (7) and (8), the analytical expressions for the spin Seebeck coefficients $\varepsilon$ and $\varepsilon_{p}$ are respectively found to be

$$
\varepsilon=\frac{\sigma_{+} \varepsilon_{+}+\sigma_{-} \varepsilon_{-}}{\sigma_{+}+\sigma_{-}} \quad \text { and } \quad \varepsilon_{p}=\frac{\sigma_{+} \varepsilon_{+}-\sigma_{-} \varepsilon_{-}}{\sigma_{+}-\sigma_{-}} .
$$

Finally, the Onsager matrix (3) can be recast as

$$
\left(\begin{array}{c}
\boldsymbol{j}_{s} \\
\boldsymbol{j} \\
\boldsymbol{j}_{p}
\end{array}\right)=-\left(\begin{array}{ccc}
\kappa & q \sigma \varepsilon & q \sigma_{p} \varepsilon_{p} \\
\sigma \varepsilon & \sigma & \sigma_{p} \\
\sigma_{p} \varepsilon_{p} & \sigma_{p} & \sigma
\end{array}\right)\left(\begin{array}{c}
\nabla T \\
\nabla V \\
\frac{1}{q} \nabla(\Delta \mu)
\end{array}\right) .
$$

3 Bulk spin current We consider an experiment where there is no electric current, i.e. $\boldsymbol{j}=0$, and a temperature gradient is imposed across the sample. In the bulk limit, i.e. $\Delta \mu=0$, the spin polarisation current density $\boldsymbol{j}_{p}$ in (10) is found to be proportional to the temperature gradient and reduces to the simple expression

$$
\dot{j}_{p}=-\sigma_{p}\left(\varepsilon_{p}-\varepsilon\right) \nabla T .
$$

This expression holds on large scales, i.e. much greater than the spin diffusion length.

The spin-dependent electric conductivities $\sigma_{+}$and the spin-dependent spin Seebeck coefficients $\varepsilon_{ \pm}$can be written as

$$
\sigma_{ \pm}=\frac{\sigma}{2}(1 \pm \beta) \quad \text { and } \quad \varepsilon_{ \pm}=\varepsilon(1 \pm \eta)
$$

Thus, to first order in $\beta$ and $\eta$, the expression (11) for the spin polarisation current density $\boldsymbol{j}_{p}$ reduces to

$$
\boldsymbol{j}_{p}=-\sigma \varepsilon(\eta-\beta) \nabla T .
$$

4 Spin Soret effect Within the two-current model, it is necessary to assume that the temperature derivative of the chemical potential is spin-dependent in order to infer the existence of a bulk spin current [12]. Instead, in the bulk limit, the three-current model shows that we can have a balance between a heat current and a spin current in the absence of a charge current, with no additional assumption.

The existence of this diffusive current is known in chemistry as a Soret effect [13]. Charles Soret observed that a tube containing a mixture of two salts, where one end of the tube was maintained cold and the other hot, presented different salt concentrations at both ends. Since then, the diffusive current of one substance with respect to another, driven by a temperature gradient, has been known as the Soret effect or thermophoresis. In liquids, it is usually demonstrated using two plates, where the top one is maintained hot and the bottom one cold in order to avoid convection. After a short while, the balance between the heat current and the diffusion flux leads the system to a stationary state [14]. Thermodiffusion is more commonly observed with aerosols in which the effect is quite intuitive: the air molecules have on average a higher velocity on the 
higher temperature side of any particle in suspension, thus pushing it to the lower temperature side.

Thus, in chemistry, the Soret effect refers to the contribution to the diffusive current of the solute with respect to the solvent [14], which is due to the temperature gradient. By analogy, the spin Soret effect refers to the contribution to polarisation current $\boldsymbol{j}_{p}^{S}$ due to the temperature gradient $\nabla T$. By defining the spin Soret coefficient $S$ as

$$
\dot{j}_{p}^{S}=-S \nabla T,
$$

it follows from (10), that the spin Soret coefficient $S$ is given by

$$
S=\sigma_{p} \varepsilon_{p} .
$$

There are many systems where two types of carriers (of charge as well as of entropy) are invoked. For example, the thermodynamical framework presented in this Letter can be generalised to a current of phonons or magnons. It has been conjectured that the difference between the effective temperature of magnons and that of phonons drives spin pumping into the electrons $[15,16]$. From a mere thermodynamic standpoint, a drift of the magnons [17] toward the cold side of the sample is a form of thermophoresis. Phonon drag can also be thought of as a cross-effect between phonons and electrons [18]. However, as always with the thermodynamics of irreversible processes, the Onsager matrix expresses the existence of relationships among the gradients of the extensive state variables and their associated currents, but cannot address the micro-mechanisms responsible for the values of the matrix elements.

5 Conclusion We consider the experimental configuration used to detect the spin Seebeck effect as one experiment characterised by zero effective electric current in the bulk limit. We show that a three-current model of heat, spin-up and spin-down currents predicts the spin polarisation current to exist on large scales and to be proportional to the temperature gradient, corresponding to a spin Soret effect. Thus, we establish the existence of a spin current driven by a heat current over large distances in the absence of a charge current and at equilibrium of the spin channels (i.e. $\boldsymbol{j}=0$ and $\Delta \mu=0$ ). Thus, a heat current may affect the magnetisation [19].

\section{References}

[1] G. E. W. Bauer, A. H. MacDonald, and S. Maekawa, Solid State Commun. 150, 459-460 (2010).

[2] M. Johnson and R. H. Silsbee, Phys. Rev. Lett. 55, 17901793 (1985)

[3] M. Johnson and R. H. Silsbee, Phys. Rev. B 35(10), 49594972 (1987).

[4] K. Uchida, S. Takahashi, K. Harii, J. Ieda, W. Koshibae, K. Ando, S. Maekawa, and E. Saitoh, Nature 455, 778-781 (2008).

[5] C. M. Jaworski, J. Yang, S. Mack, D. D. Awschalom, J. P. Heremans, and R. C. Myers, Nature Mater. 9, 898-903 (2010).

[6] K. Uchida, H. Adachi, T. An, T. Ota, M. Toda, B. Hillebrands, S. Maekawa, and E. Saitoh, arXiv:1103.6120v1 (March 2011).

[7] L. Gravier, S. Serrano-Guisan, F. Reuse, and J. Ansermet, Phys. Rev. B 73(2), 024419 (2006).

[8] A. Slachter, F. L. Bakker, J. Adam, and B. J. van Wees, Nature Phys. 6, 879-882 (2010).

[9] T. Valet and A. Fert, Phys. Rev. B 48(10), 7099-7113 (1993).

[10] J. P. Ansermet, IEEE Trans. Magn. 44(3), 329-335 (2008).

[11] O. Tsyplyatyev, O. Kashuba, and V. I. Fal'Ko, Phys. Rev. B 74(13), 132403 (2006).

[12] K. Uchida, S. Takahashi, J. Ieda, K. Harii, K. Ikeda, W. Koshibae, S. Maekawa, and E. Saitoh, J. Appl. Phys. 105(7), 070000 (2009).

[13] C. Soret, Archives des Sciences Physiques et Naturelles (Genève) 2, 48-61 (1879).

[14] D. D. Fitts, Nonequilibrium Thermodynamics (McGrawHill, Colombus, 1962).

[15] K. Uchida, J. Xiao, H. Adachi, J. Ohe, S. Takahashi, J. Ieda, T. Ota, Y. Kajiwara, H. Umezawa, H. Kawai, G. E. W. Bauer, S. Maekawa, and E. Saitoh, Nature Mater. 9, 894897.

[16] J. Xiao, G. E. W. Bauer, K. Uchida, E. Saitoh, and S. Maekawa, Phys. Rev. B 81(21), 214418 (2010).

[17] Y. Kajiwara, K. Harii, S. Takahashi, J. Ohe, K. Uchida, M. Mizuguchi, H. Umezawa, H. Kawai, K. Ando, K. Takanashi, S. Maekawa, and E. Saitoh, Nature 464, 262-266 (2008).

[18] A. Miele, R. Fletcher, E. Zaremba, Y. Feng, C. T. Foxon, and J. J. Harris, Phys. Rev. B 58, 13181-13190 (1998).

[19] M. Hatami, G. E. W. Bauer, Q. Zhang, and P. J. Kelly, Phys. Rev. B 79(17), 174426 (2009). 